\title{
CYBORG-The Smart Home Assistance Robot
}

\author{
Anupama Thomas ${ }^{1}$, Georgina Binoy Joseph ${ }^{2}$, Manu Augustine ${ }^{3}$ \\ M Tech VLSI and Embedded System Design Student, ECE Dept, TIST, Arakunnam, Ernakulam, Kerala ${ }^{1}$ \\ Dept of ECE, Faculty of Engineering, TIST, Arakunnam, Ernakulam, Kerala ${ }^{2}$ \\ Arvin Technologies, Kalamassery, Ernakulam, Kerala ${ }^{3}$
}

\begin{abstract}
The field of socially assistive robotics is growing but has not yet been properly defined and circumscribed. There has been significant attention given to and great progress made in contact assistive robotics. Currently, there is no clear definition of robots that provide assistance through interaction and without physical contact, namely socially assistive robotics. Assistive robots in smart home environments have been suggested as possible cost-effective care solutions to demographic changes characterized by an increasingly elderly population. The vision is that service robots are available in the home to help and assist elderly residents. This paper proposes a smart home companion robot CYBORG. This robot can be used to save power to switch off unnecessary lights. It can be used as an alarm to remind the family members about important activities. It will also help people clean their homes and water plants. The key feature is that the interface for all the functions will be simple allowing elderly people or their relatives make the robot learn activities in the smart home and teach it to carry out behaviours in response to these activities. This mechanism provides support, assistance and active engagement over time along with personalization.
\end{abstract}

Keywords: Assistive Robot, CYBORG, Smart Home, personalization.

\section{INTRODUCTION}

Socially intelligent robotics is the way of creating robots capable of exhibiting natural appearing social qualities. Besides the basic capabilities of moving and acting autonomously, the field has focused on the use of the robots to communicate and interact with users to provide support. Socially assistive robots focus on helping human users through socially and also by physical interaction. The study of human robot interaction (HRI) for socially assistive robotics applications is a new, interdisciplinary an increasingly popular research area that brings together robotics, medicine, social and cognitive sciences, and neuroscience. Assistive robotics in general and socially assistive robotics, in particular, is an important field that helps to improve the quality of life for broad populations of users.

Assistive robots in smart-home environments have been suggested as a possible cost and care solution to an increasingly elderly population. The vision is that assistive robots are available to help and assist elderly people. This mechanism provides support, assistance, and active engagement over time. Achieving personalization presents several challenges for a companion robot. Simple scripting of interactions will not reach the above aims due to the dynamics of the interaction and the key requirement of the robot to develop and learning is necessary. The robot often uses a vision system or some other sensory modality (e.g., infrared sensors, electronic markers) to observe the presence of the user and perform its functions.

An assistive robot must effectively interact with people it is serving. The communication between the human and robot is possible by using different means: a speech interface method, a sensor, a touch-screen/keypad, and human gesture.
Verbal and non-verbal communication plays a crucial role in socially assistive robotics and provides social cues that make the robot appear more intuitive and natural to the user. Robots must maintain an appropriate spatial distance to people so as to respect their personal and social spaces. In order to engage in social interactions, the robot needs to be aware of human presence, detect the willingness of humans to interact, and determine and learn appropriate personal space ranges for various users. The robots must be endowed with human-oriented interaction skills and capabilities to learn from us or to teach us, as well as to communicate with us and understand us. The different user groups responses, gender, and domain differences should be evaluated accurately. Another very important challenge is to make safe robots that can be easily operated by all age groups.

The paper is structured as follows: Related works are depicted in section II. The control system implemented is described in section III. The hardware description is given in section IV. The conclusions drawn are presented in section $\mathrm{V}$.

\section{RELATED WORKS}

The assistive robot is not a new concept. Each design has specific manufacturing methods, advantages and disadvantages. But all these designs are not human friendly. By reviewing different papers and an assistive robot which is based on ARM controller was developed.

An Intelligent Home Environment Inspecting Robot [1] was developed in 2007 by Ruijie Zhang et al. It is the one which can inspect home environment and give necessary information to the residents. It is a three-wheeled mobile 
Vol. 4, Issue 2, February 2017

robot which consists of several types of sensors. It gives information about room temperature and also detects dangerous gas and fire, and gives alarm through voice, short message and display on a remote computer by a wireless network.

H.-M. Gross et al developed a robot in 2012 which can be used by people with Mild Cognitive Impairment[2]. This robot is meant to support the elderly people in scheduling their tasks, reminding to take their medicine, or doing a video/telephony call. It can be controlled remotely or drive autonomously to give the exact location.

In 2013, a Humanoid Robot which is able to control home appliances and systems was proposed by Thirukural.R et al[3]. Home automation is the most demanding requirement in today's fast moving lifestyle. This system is able to remotely access the home appliances using a Humanoid Robot, Nao. The proposed framework consists of two modules communication and control module. Communication module uses Team Viewer software to provide remote access. The control module controls the appliances using the IR sensor present in Nao. However, in view of compatibility issues, alternate object recognition, and a grasping module are used. Image processing algorithm is developed in Nao to detect the remote object.

David Portugal et al developed a social robot in 2015. This mobile robot platform has been integrated with virtual social care technology to meet the requirements of elderly people, following a human centred approach. From all these studies it can be noted that there are several techniques and applications involved in the case of assistive robots[5].

Existing research on data collection about assistive robots came to a conclusion that there are some disadvantages. Also, technical skills are required to operate this system. So to avoid these problems a new model is developed. So considering these applications, CYBORG is made. It allows the house resident to personalize the robot to meet their changing needs.

\section{III.CONTROL SYSTEM}

CYBORG can be used as a power saver- to switch off unnecessary lights and fans. The robot device includes a person detection unit that detects the presence of a person in the external environment. It can be used as an alarm to remind the family members about important activities and can also help clean the home. This system interfaces with the smart home, allowing elderly people or their relatives to use it efficiently. A two-wheeled drive is used for locomotion.

The system uses a PIR sensor to detect the presence of a human in the room and using ZIGBEE communication; the system turns the light and fan on/off accordingly.

An LPG sensor is used to detect gas leakage and alert will be given if detected. A vacuum cleaner facility is incorporated to clean the house. The robot movement is through line follower method. An IR sensor is used to detect the obstacles. When the system detects any obstacle, the buzzer beeps a sound. Real Time Clock (RTC) is used to set a particular date and time to remind about medicines, important event and also to set alarm. An LCD is used to display these data.

A.ARM Microcontroller

The controlling unit used is the LPC21xx series ARM microcontroller. Especially LPC2148 is chosen. CPU operating voltage range is $3.3 \mathrm{~V}$. The controller coordinates all the functions that are to be performed. It is based on RISC Architecture. It has so many features like 8 to $40 \mathrm{kB}$ static RAM, one or two 10 bit ADC, a DAC, two timers/counters, up to 45 GPIO pins and low power realtime clock.

\section{B. Vacuum Cleaning Unit}

The robot navigates within the room using a line follower method and identifies the obstacles using IR sensor. CYBORG has a vacuum cleaner within the system. When a switch is pressed, the vacuum cleaner will be on and the robot goes along the desired path to clean the house.

\section{PIR Sensor}

For energy efficient and controlled lighting system, human localization is necessary. A PIR sensor is used to sense motion and is used to detect whether a human has moved in or out of the sensors range. When the robot detects human presence, it will turn the on the light and fan and vice versa.

\section{Reminder Unit}

A major challenge faced by many elderly is a decline in cognitive functioning; particularly in memory.Task management is another important concern. CYBORG gives support for this. This section mainly consists of RTC and alarm. The purpose of an RTC or a real time clock is to provide precise time and date which can be used for various applications.

\section{E. LPG Sensor}

LPG sensor is used to detect the presence of dangerous gases. The sensor MQ5 can detect the gas concentration ranging from 200 to $10000 \mathrm{ppm}$. Beyond the threshold, an alert will be given by the robot.

\section{HARDWARE DESCRIPTION}

The system is implemented using ARM Microcontroller as the base microcontroller. The ARM shield is provided with PIR sensor, LPG sensor and keypad to specify time as inputs. The block diagram is shown in fig. 1 .

Since the tasks do not depend on each other, this board is capable of doing all tasks in parallel, thus providing a faster response for the system. An LCD unit is present to display the requirements. The vacuum cleaner unit has a relay that gets energised when a switch is pressed turning on the vacuum cleaner.

The output of the PIR section is sent to the power section via ZIGBEE which gives information about the presence 
Vol. 4, Issue 2, February 2017

of a person in the room. Users can manually set the date and time via a keypad for the RTC section. When a particular set time is reached the alarm will sound. LPG sensor is also present to detect the presence of any gas leakage in the house. The movement of the robot is controlled by two motors. The requirements are displayed on an LCD Display.

The system consists of a main block and a power section. The main block is present inside the mobile part, while the power section is present near the switch board. The main block consists of different sensors, LCD display unit, ZIGBEE unit, Vacuum cleaner, RTC and a buzzer. The power section consists of a relay driver, ZIGBEE, and a display unit.

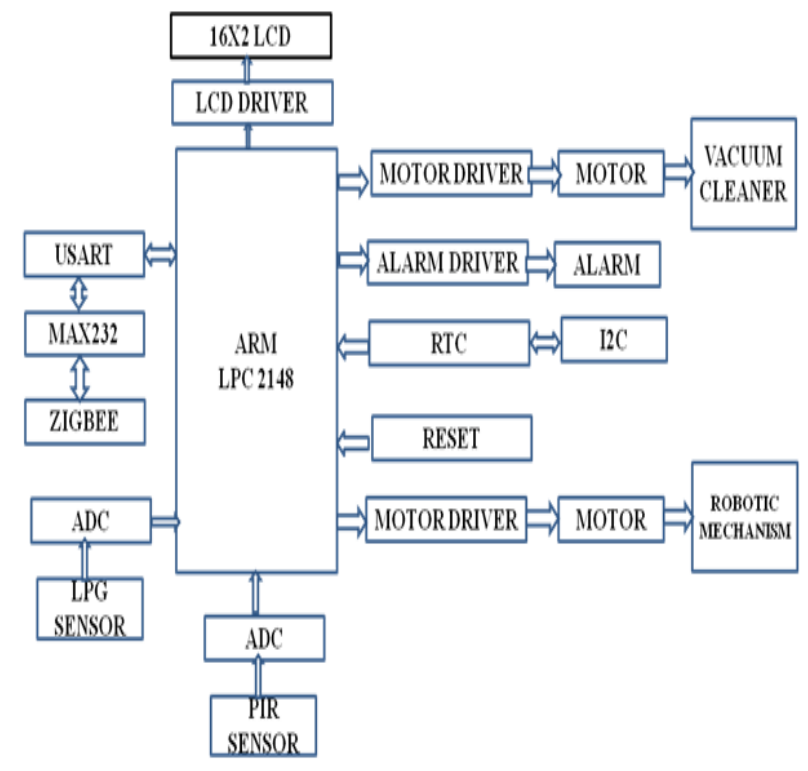

Fig.1 Block Diagram - Main Block

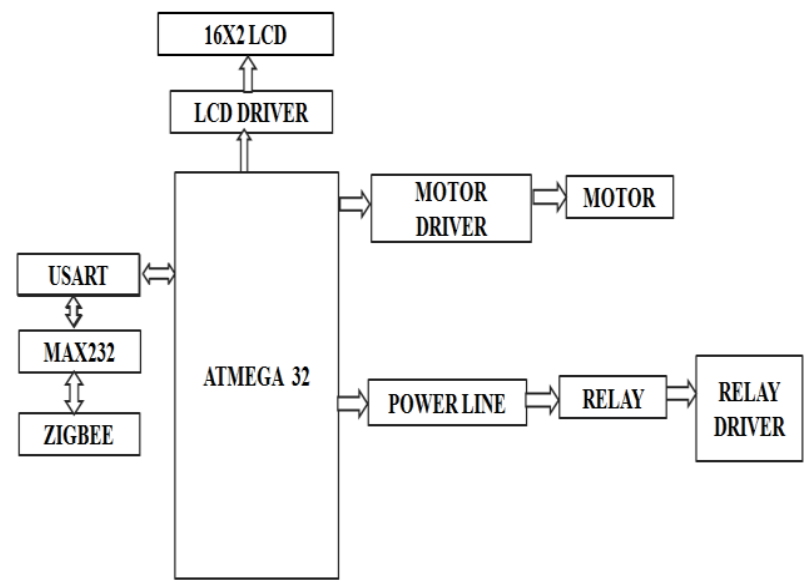

Fig.2 Block Diagram - Power Section

The message sends from the main block via ZIGBEE is received by the ZIGBEE module of the power section. If the message received is that no one is there in the room, and then the relay cuts the power line, else all the lights and fans will remain on. The proposed system is shown in Fig.3. The two boards are shown here.

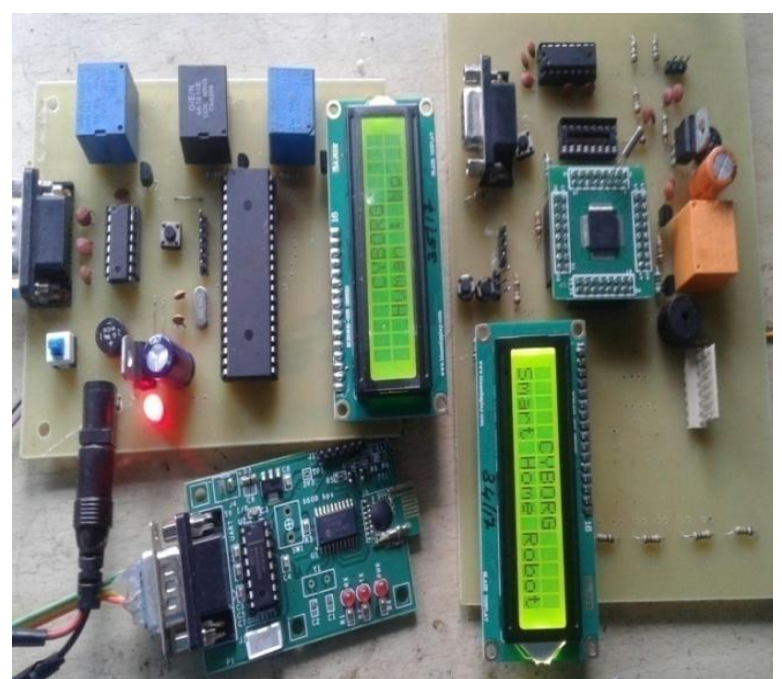

Fig. 3 Hardware setup of the proposed system.

\section{CONCLUSION}

CYBORG is a robot personalization system designed to be used by persons operating in assistive environments in smart homes, typically carers, relatives or the elderly person themselves. Considering the previous systems it has a large number of benefits. This robot can be used to save power to switch off unnecessary lights.

Another feature is that it can be used as an alarm to remind the family members about important activities. It will also help people clean the home. The key feature is that the interface for all the functions will be simple allowing elderly people or their relatives to do the activities in the smart home and carry out behaviours in response to these activities.Several future directions exist to enhance CYBORG including the use of solar power to activate the system and the addition of a face recognition system.

\section{REFERENCES}

11] Ruijie Zhang, Fujun He, Zhijiang Du and Lining Sun, An Intelligent Home Environment Inspecting Robot, in Proceedings of the 2007 IEEE International Conference on Mechatronics and Automation August 5 - 8, 2007, Harbin, China

[2] H.-M. Gross, Ch. Schroeter, S. Mueller A. Bley, T. Langner C. Huijnen, H. van den Heuvel, Further Progress towards a Home Robot Companion for People with Mild Cognitive Impairment 2012 IEEE International Conference on Systems, Man, and Cybernetics

[3] Thirukural.R, Pradeep.M, Sengottuvel.K and Bhuvaneswari.P.T.V, Remote Access of Home Appliances Using Humanoid Robot, 2013 International Conference on Recent Trends in Information Technology (ICRTIT) October 14-17, 2012, COEX, Seoul, Korea

[4] Mohammed El-Shafei,Adel Al Shalati and Mher Rehayel ,"HOBOT: A customizable home management system with a surveillance RoBOT", applying self-adaptation technologies,

[5] David Portugal, Lus Santos, Paulo Alvito, Jorge Dias, George Samaras and Eleni Christodoulou,"SocialRobot: An Interactive Mobile Robot for Elderly Home Care", IEEE/SICE International Symposium on System Integration (SII) December 11-13, 2015.

[6] Joe Saunders, Dag Sverre Syrdal, Kheng Lee Koay, Nathan Burke, and Kerstin Daut-enhahn,"Teach MeShow MeEnd-User Personalization of a Smart Home and Companion Robot",IEEE Transactions On Human-Machine Systems, VOL. 46, NO. 1, FEBRUARY 2016 